Case Report

\title{
Unusual Ventilatory Response to Exercise in Patient with Arnold-Chiari Type 1 Malformation after Posterior Fossa Decompression
}

\author{
Keely Smith,, ${ }^{1}$ Ana M. Gomez-Rubio, ${ }^{1}$ Tomika S. Harris, ${ }^{1}$ \\ Lauren E. Brooks, ${ }^{2}$ and Ricardo A. Mosquera ${ }^{1}$ \\ ${ }^{1}$ Department of Pediatrics, McGovern Medical School, University of Texas Health Science Center at Houston, \\ Houston, TX 77030, USA \\ ${ }^{2}$ McGovern Medical School, University of Texas Health Science Center at Houston, Houston, TX 77030, USA \\ Correspondence should be addressed to Ricardo A. Mosquera; ricardo.a.mosquera@uth.tmc.edu
}

Received 15 April 2016; Accepted 26 May 2016

Academic Editor: Piero Pavone

Copyright (C) 2016 Keely Smith et al. This is an open access article distributed under the Creative Commons Attribution License, which permits unrestricted use, distribution, and reproduction in any medium, provided the original work is properly cited.

\begin{abstract}
We present a case of a 17-year-old Hispanic male with Arnold-Chiari Type 1 [AC-Type 1] with syringomyelia, status post decompression, who complains of exercise intolerance, headaches, and fatigue with exertion. The patient was found to have diurnal hypercapnia and nocturnal alveolar hypoventilation. Cardiopulmonary testing revealed blunting of the ventilatory response to the rise in carbon dioxide $\left(\mathrm{CO}_{2}\right)$ resulting in failure of the parallel correlation between increased $\mathrm{CO}_{2}$ levels and ventilation; the expected vertical relationship between $\mathrm{PETCO}_{2}$ and minute ventilation during exercise was replaced with an almost horizontal relationship. No new pathology of the brainstem was discovered by MRI or neurological evaluation to explain this phenomenon. The patient was placed on continuous noninvasive open ventilation (NIOV) during the day and CPAP at night for a period of 6 months. His $\mathrm{pCO}_{2}$ level decreased to normal limits and his symptoms improved; specifically, he experienced less headaches and fatigue during exercise. In this report, we describe the abnormal response to exercise that patients with AC-Type 1 could potentially experience, even after decompression, characterized by the impairment of ventilator response to hypercapnia during exertion, reflecting a complete loss of chemical influence on breathing with no evidence of abnormality in the corticospinal pathway.
\end{abstract}

\section{Introduction}

Arnold-Chiari Type 1 (AC-Type 1) malformation is a complex syndrome in which the brainstem, medulla, and cerebellar tonsils herniate through the posterior fossa into the cervical spinal canal. Compression of the brainstem structures, including the respiratory center and its neural circuits, results in a wide variety of symptoms related to breathing such as sleep hypoventilation, central sleep apnea, and respiratory failure [1]. Medical literature suggests that most respiratory symptoms related to AC-Type 1 resolve after surgical decompression of the foramen magnum $[1,2]$. However, in spite of surgical intervention, some patients continue to experience adverse respiratory symptoms [3].
During normal exercise, there is a physiologic increase in both oxygen consumption and carbon dioxide $\left(\mathrm{CO}_{2}\right)$ production until the anaerobic threshold, which is typically about $60 \%$ of the subject's maximum work capacity, is reached. At this point, anaerobic metabolism leads to an increase in $\mathrm{CO}_{2}$ production compared to oxygen consumption. However, the arterial $\mathrm{pCO}_{2}$ level remains constant because the increase of $\mathrm{CO}_{2}$ stimulates central chemoreceptors in the medulla, triggering an increase in ventilation, thereby preventing hypercapnia $[4,5]$.

In this case report, we present a patient with AC-Type 1 with syringomyelia complaining of fatigue, headache, and somnolence during and after exercise. In addition, impairment of ventilator response to hypercapnia (baseline 60-70) 
during exertion was shown, reflecting a complete loss of chemical influence on breathing with no evidence of abnormality in the corticospinal pathway.

\section{Case Presentation}

A 17-year-old Hispanic male with a history of multiple medical and surgical problems associated with AC-Type 1 malformation and syringomyelia, status after surgical decompression, was referred to our Pulmonary Clinic for evaluation of respiratory symptoms. He presented with symptoms of exercise intolerance characterized by headaches, fatigue, and somnolence during and after exercise. In addition, he reported chronic symptoms of fatigue, irritability, increased daytime somnolence, early morning headaches, and disordered sleep breathing.

A thorough evaluation was performed. Arterial blood gas revealed the following results: $\mathrm{pH}$ of $7.31, \mathrm{pCO}_{2}$ of $63 \mathrm{mmHg}$, $\mathrm{pO}_{2}$ of $93, \mathrm{HCO}_{3}$ of $32 \mathrm{mMol} / \mathrm{L}$, and a normal $\mathrm{O}_{2}$ saturation at $96 \%$. Nocturnal polysomnography demonstrated end-tidal $\mathrm{CO}_{2}$ as high as $61 \mathrm{mmHg}$ and $\mathrm{CO}_{2}$ for greater than 50 torr for 99\% of total sleep time (tst), no apnea, and 13 hypopneas/hr. Spirometry at rest showed normal values: Forced-Volume Capacity (FVC) 117\%, forced-expiratory volume 1 second (FEV1) 121\%, FEV1/FVC 104\%, and forced-expiratory flow (FEF) Max $115 \%$.

The patient underwent a symptom-limited incremental cardiopulmonary exercise test (CPET). The patient was exercised on an electronically braked cycle ergometer using 20watt incremental protocol while heart rate, blood pressure, tidal volume, minute ventilation, oxygen saturation, oxygen consumption, $\mathrm{CO}_{2}$ production, and perceptive response to exercise were monitored.

At baseline, the following values were recorded: respiratory rate $(\mathrm{RR})$ was $10 \mathrm{BPM}$, tidal volume $\left(V_{T}\right)$ was $0.57 \mathrm{Lm}$, minute ventilation (Ve) was $5.9 \mathrm{~L} / \mathrm{min}, \mathrm{SpO}_{2}$ was $96 \%, \mathrm{VO}_{2}$ was $3.8 \mathrm{~mL} / \mathrm{kg} / \mathrm{min}$, heart rate (HR) was $97 \mathrm{bpm}$, blood pressure (BP) was 105/63, and $\mathrm{PETCO}_{2}$ was $45 \mathrm{mmHg}$ (Table 1). After exercising for 9 minutes, the patient achieved anaerobic threshold, at which point the partial pressure of exhaled carbon dioxide $\left(\mathrm{PETCO}_{2}\right)$ increased to $55 \mathrm{mmHg}$ but Ve increased to only $31.5 \mathrm{~L} / \mathrm{min}$. The patient reached $\mathrm{VO}_{2} \mathrm{Max}$ at 12 minutes after starting exercise, while working at a rate of 177 watts and a speed of $66 \mathrm{RPM}$, at which point the $\mathrm{PETCO}_{2}$ remained at $55 \mathrm{mmHg}$ and Ve increased to $79.9 \mathrm{~L} / \mathrm{min}(50 \%$ of predicted value).

The test also revealed normal values with regard to heart rate and rhythm, oxygen pulse response, and blood pressure. No abnormalities of heart rhythm or ST segment were noted and the patient did appear to have a low aerobic capacity and normal exercise efficiency. His maximum exercise was not limited by ventilation, and his breathing reserve was within normal values.

Evaluation by a neurologist revealed normal deep tendon reflexes, gait, and motor tone, ruling out suspicions of corticospinal tract abnormality. Additionally, his MRI was normal, showing that his brainstem structures were no longer compressed.
TABLE 1: Cardiopulmonary exercise test results.

\begin{tabular}{lccc}
\hline & Baseline & $\begin{array}{c}\text { Anaerobic } \\
\text { threshold }\end{array}$ & $\mathrm{VO}_{2} \mathrm{Max}$ \\
\hline Ventilation & & & \\
$\mathrm{RR}$ & $10 \mathrm{BPM}$ & $15 \mathrm{BPM}$ & $31 \mathrm{BPM}$ \\
$V_{T}$ & $0.57 \mathrm{~L}$ & $2.13 \mathrm{~L}$ & $2.56 \mathrm{~L}$ \\
$\mathrm{Ve}$ & $5.9 \mathrm{~L} / \mathrm{min}$ & $31.5 \mathrm{~L} / \mathrm{min}$ & $79.9 \mathrm{~L} / \mathrm{min}$ \\
$\mathrm{SpO}_{2}$ & $96 \%$ & $96 \%$ & $96 \%$ \\
\hline $\mathrm{O}_{2} \mathrm{consumption}$ & & & \\
$\mathrm{VO}_{2}(\mathrm{~mL} / \mathrm{kg} / \mathrm{min})$ & 3.8 & 22.7 & $34.6(78 \% \mathrm{predicted})$ \\
$\mathrm{VCO}_{2}$ & 210 & 1671 & 3403 \\
$\mathrm{RER}$ & 0.81 & 1.08 & 1.43 \\
\hline $\mathrm{Cardiac}$ & & & \\
$\mathrm{HR}^{\mathrm{VQ}}$ & $97 \mathrm{bpm}$ & $158 \mathrm{bpm}$ & $194 \mathrm{bpm}$ \\
\hline $\mathrm{Ve} / \mathrm{VCO}_{2}$ & 28 & 19 & 23 \\
$\mathrm{Ve} / \mathrm{VO}_{2}$ & 23 & 20 & 34 \\
$\mathrm{PETCO}_{2}$ & $45 \mathrm{mmHg}$ & $56 \mathrm{mmHg}$ & $50 \mathrm{mmHg}$ \\
$\mathrm{PETO}$ & $93 \mathrm{mmHg}$ & $91 \mathrm{mmHg}$ & $109 \mathrm{mmHg}$ \\
\hline
\end{tabular}

The patient was treated with 6 months of noninvasive open ventilation (NIOV) during the day and was placed on CPAP at night. NIOV was discontinued after 6 months of treatment, and his $\mathrm{pCO}_{2}$ was obtained. At this point, his venous $\mathrm{pCO}_{2}$ was within the normal limit $(50 \mathrm{mmHg})$. Two months after NIOV was discontinued, the $\mathrm{pCO}_{2}$ remained normal $(42 \mathrm{mmHg})$. In addition, the cardiopulmonary test was repeated and showed a slight improvement in ventilatory response. Specifically, the $\mathrm{PETCO}_{2}$ started at $41 \mathrm{mmHg}$ and stayed at $41 \mathrm{mmHg}$ at peak exercise $\left(\mathrm{VO}_{2} \mathrm{Max}\right)$. An increase in $\mathrm{Ve}$ and $\mathrm{Ve} / \mathrm{VCO}_{2}$ was also observed compared to before treatment.

\section{Discussion}

We present a teenage male with AC-Type 1 with exerciseinduced hypercapnia after decompressive surgery, who was treated with continuous noninvasive open ventilation (NIOV) for a period of 6 months in order to reset the respiratory central chemoreceptors. Treatment with NIOV improved both the diurnal and exercise-induced hypercapnia.

Individuals with Arnold-Chiari Type 1 could potentially have blunted ventilatory response to hypercapnia and hypoxemia, which suggests abnormalities of central chemoreceptors. The cause of central hypoventilation in patients with Chiari malformation is thought to be due to dysgenesis of neural structures or damage to the brainstem and cerebellum during the herniation, causing impairment of the respiratory centers [6]. However, the symptoms of diurnal and exerciseinduced hypercapnia, sleep disordered breathing, and vocal cord dysfunction persisted in our patient, despite successful surgical decompression and normal MRI. 


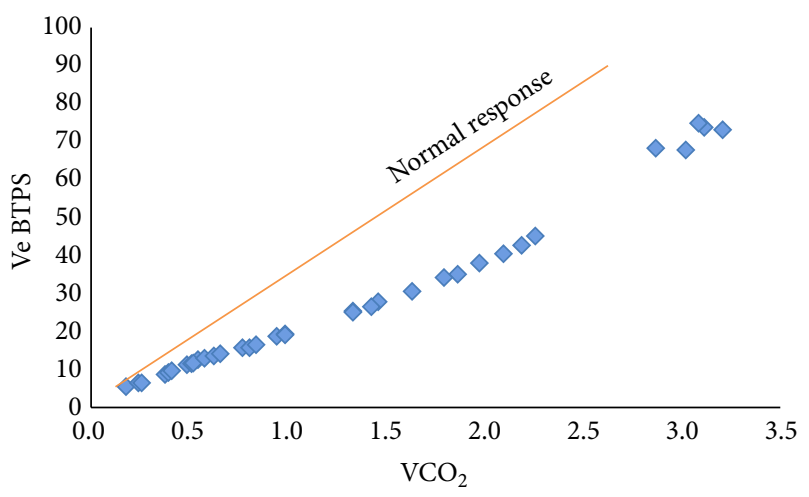

FIGURE 1: Relationship between $\mathrm{Ve}$ and $\mathrm{VCO}_{2}$ before treatment.

The ventilatory response to constant work-rate exercise consists of 3 phases involving multiple mechanisms. The ventilatory response that occurs at the beginning of exercise is characterized by an immediate increase in ventilation. The next phase consists of a slow increase in ventilation, ultimately reaching a final steady phase if the exercise is not too severe. Ventilatory parameters in this phase show an increase in minute ventilation [Ve] due to both tidal volume $\left[V_{T}\right]$ and respiratory rate $[R R]$. Initially, the increase in $V_{T}$ exceeds the respiratory rate. However, as metabolic acidosis develops, the respiratory rate predominates. The mechanism by which minute ventilation increases during this phase remains controversial. At mild to moderate exercise, characterized by exercise levels not reaching lactic acidosis, there is an increase in ventilation primarily driven by arterial chemoreceptors which respond to oscillatory changes in the blood gases, despite the $\mathrm{PaCO}_{2}$ and $\mathrm{PaO}_{2}$ levels [5].

In our patient, the level of $\mathrm{PETCO}_{2}$ significantly increased during exercise. In addition, Ve did not increase as expected most likely due to an abnormality in the central chemoreceptors, which is associated with lower Ve (Figure 1). It is known that, in patients with progressive neuromuscular degenerative disease, respiratory compromise leads to disordered breathing and chronic hypercapnia, which may be alleviated by resetting the sensitivity of the central respiratory centers with noninvasive open ventilation for at least four hours a day [7]. With this concept in mind, the patient was placed on 6 months of NIOV which improved both his baseline $\mathrm{pCO}_{2}$ and his exercised induced hypercapnia. These effects persisted even 2 months after NIOV was discontinued. We speculate that the respiratory central chemoreceptors were reset.

This case underscores the importance of a thorough evaluation of the respiratory drive, including cardiopulmonary exercise testing, in patients with AC-Type 1 with chronic symptoms of exercise intolerance including headaches, fatigue, somnolence, and hypercapnia.

\section{Competing Interests}

The authors declare that there are no competing interests regarding the publication of this paper.

\section{References}

[1] A. Imperato, V. Seneca, V. Cioffi, G. Colella, and M. Gangemi, "Treatment of Chiari malformation: who, when and how," Neurological Sciences, vol. 32, no. 3, pp. S335-S339, 2011.

[2] J. Klekamp, U. Batzdorf, M. Samii, and H. W. Bothe, "The surgical treatment of Chiari I malformation," Acta Neurochirurgica, vol. 138, no. 7, pp. 788-801, 1996.

[3] P. Haouzi, J.-C. Marchal, E. M. Allioui, B. Hannhart, B. Chalon, and M. Braun, "Corticospinal pathway and exercise hyperpnea: lessons from a patient with Arnold Chiari malformation," Respiration Physiology, vol. 123, no. 1-2, pp. 13-22, 2000.

[4] J. Duffin, "The role of the central chemoreceptors: a modeling perspective," Respiratory Physiology \& Neurobiology, vol. 173, no. 3, pp. 230-243, 2010.

[5] K. Wasserman, "Breathing during exercise," The New England Journal of Medicine, vol. 298, no. 14, pp. 780-785, 1978.

[6] C. M. Cielo and C. L. Marcus, "Central hypoventilation syndromes," Sleep Medicine Clinics, vol. 9, no. 1, pp. 105-118, 2014.

[7] H. B. Panitch, "Diurnal hypercapnia in patients with neuromuscular disease," Paediatric Respiratory Reviews, vol. 11, no. 1, pp. $3-8,2010$. 


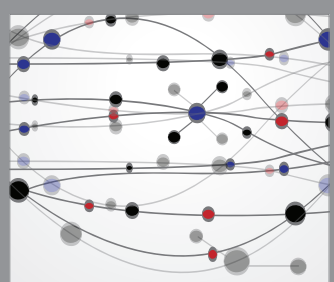

The Scientific World Journal
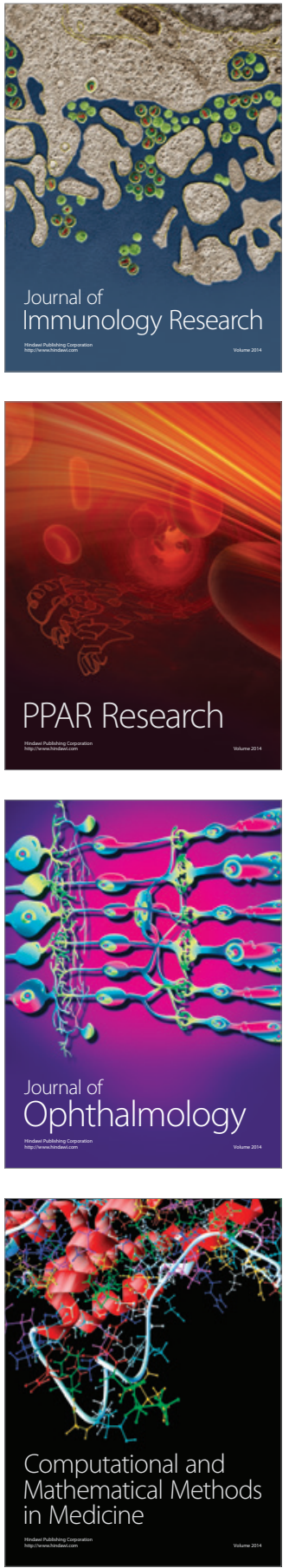

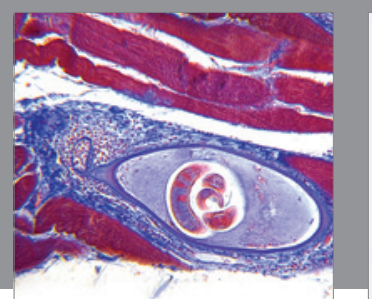

Gastroenterology Research and Practice

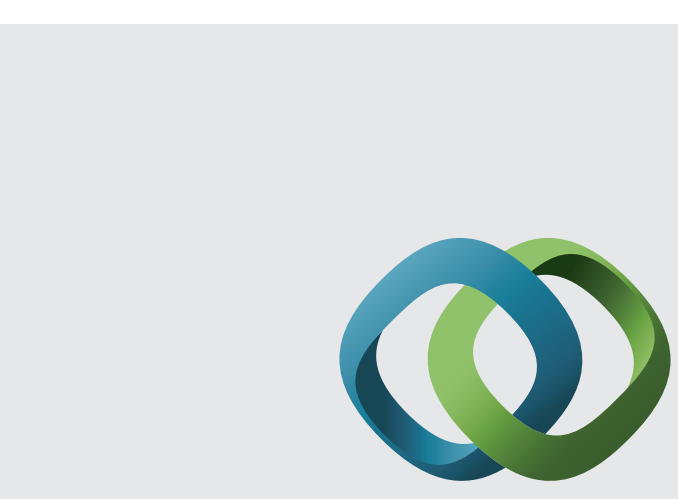

\section{Hindawi}

Submit your manuscripts at

http://www.hindawi.com
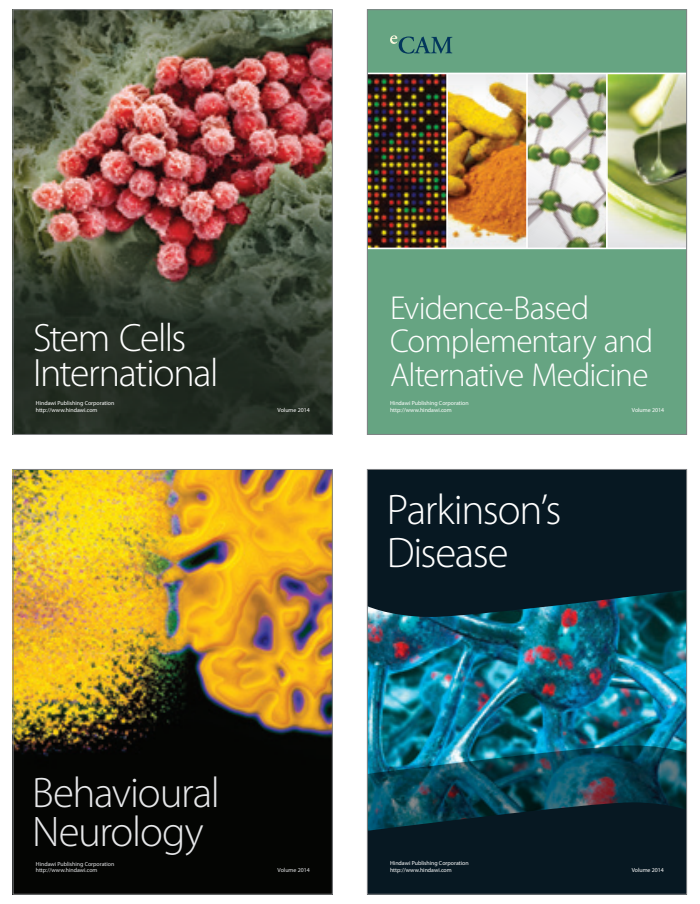
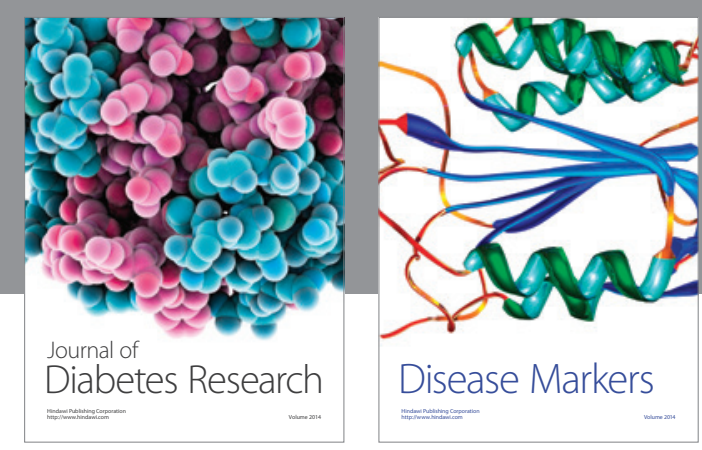

Disease Markers
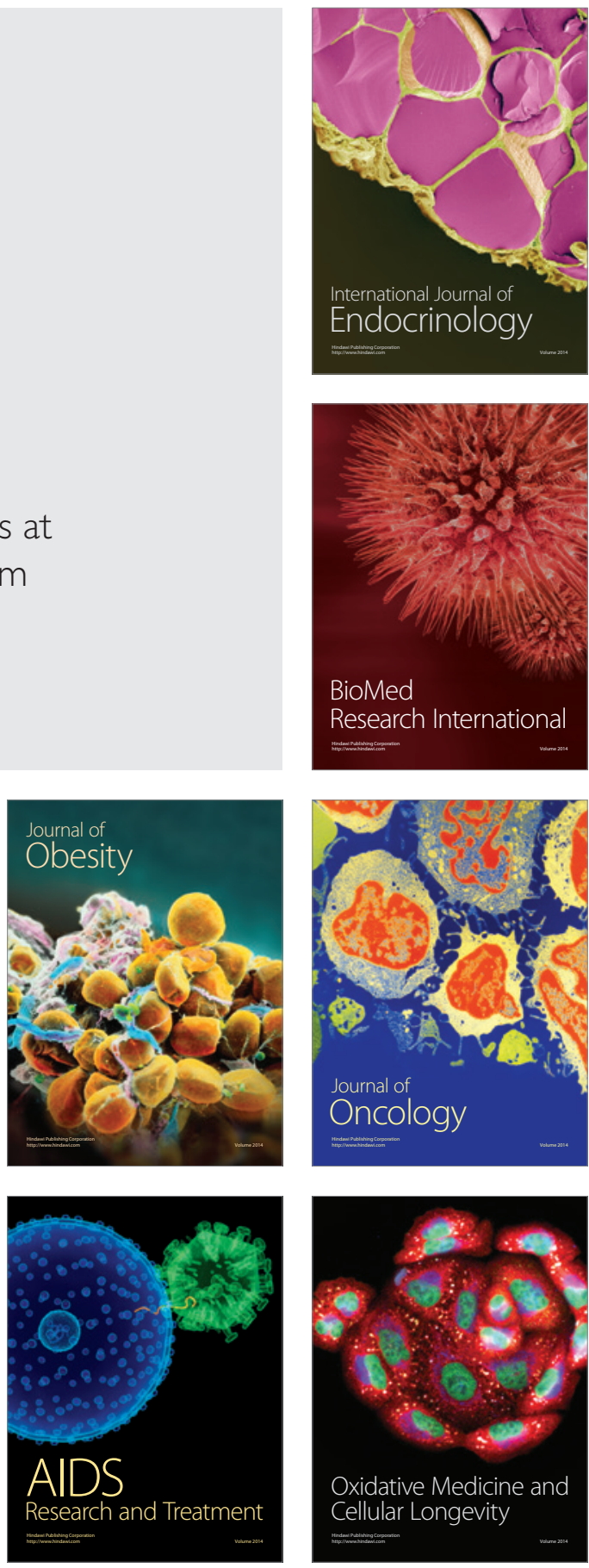